\title{
Reciclaje de la escoria negra para su uso como materia prima en el sector de la construcción. Caso de estudio ANDEC
}

Recycling of black slag for use as raw material in the construction sector. Case study ANDEC

Mgs, Mariana de Jesús Alvarado Márquez

Fecha de recepción: 5 de noviembre de 2018

Fecha de aceptación: 20 de diciembre de 2018 


\title{
Reciclaje de la escoria negra para su uso como materia prima en el sector de la construcción. Caso de estudio ANDEC
}

Recycling of black slag for use as raw material in the construction sector. Case study ANDEC

Mariana Alvarado Márquez ${ }^{1}$

Como citar: Alvarado, M. (2019). Reciclaje de la escoria Negra para uso como materia prima en el sector de la construcción. Caso de estudio ANDEC, Revista Universidad de Guayaquil. 128(1), 34-44.DOI: https://doi.org/10.53591/rug.v128i1.1389

\section{RESUMEN}

El presente estudio tiene como objetivo identificar la importancia de la escoria negra como materia prima o componente en los sectores de la construcción, a través del conocimiento del perfil de los clientes del sector y su percepción del uso de la escoria para este importante sector de la economía. Para ello se realizó una investigación cualitativa y cuantitativa la cual permitió determinar el uso de la escoria para obras menores y adecuaciones dentro de la empresa ANDEC y emprender un proyecto social utilizando este producto sin fines de lucro, para el arreglo de calles, rellenos o construcciones menores para la comunidad, creando una percepción positiva dentro de la misma acerca del uso de la escoria para aplicaciones de la construcción.

\section{PALABRAS CLAVE}

Escoria, Acero, construcción.

\begin{abstract}
The present study aims to identify the importance of black slag as a raw material or component in the construction sectors, through knowledge of the profile of the sector's clients and their perception of the use of slag for this important sector of the economy. For this, a qualitative and quantitative investigation was carried out which allowed to determine the use of slag for minor works and adjustments within the company ANDEC and to undertake a social project using this non-profit product, for the repair of streets, landfills or minor construction for the community, creating a positive perception within the community about the use of slag for construction applications.
\end{abstract}

\section{KEYWORDS}

Slag, Steel, construction.

\footnotetext{
${ }^{1}$ Magister en Finanzas y Proyectos Corporativos, Investigador independiente, Ecuador. Correo electrónico: mariana-am@hotmail.es
}

Esta obra está bajo una licencia internacional Creative Commons Atribución-NoComercial-SinDerivadas 4.0. Los autores mantienen los derechos sobre los artículos y por tanto son libres de compartir, copiar, distribuir, ejecutar y comunicar públicamente la obra. 


\section{INTRODUCCIÓN}

ANDEC es una de las principales empresas siderúrgicas en el Ecuador con casi seis décadas de trayectoria empresarial, proporcionando el acero para la construcción. ANDEC es una empresa fabricante de productos de acero para refuerzo de hormigón y otros perfiles livianos. (Jiménez \& Navarrete, 2018) Para cumplir con este objetivo la empresa se encuentra conformada por 3 procesos industriales, los cuales se dividen en: Captación y procesamiento de chatarra, Acería y Laminación. (ANDEC, 2018)

La escoria siderúrgica se genera como el principal residuo del proceso de acería, esta se forma al momento al agregar aditivos como cal, manganeso, silicio, aluminio, carbono y oxigeno durante la fundición de la chatarra o afinación del acero líquido, debido a esto se forma una espuma que contiene todos los remanentes en forma de óxidos, sulfuros y metales. (Nicolalde, 2008, pág. 18) Se ubica en la parte superficial del acero líquido, esta espuma que es la escoria en general tiene la función de eliminar residuos en el acero líquido para lograr la composición química requerida por la norma, también se utiliza para mantener la temperatura y evitar el ingreso al acero líquido de impurezas y gases durante la fundición.

En el proceso acería se puede conseguir 2 tipos de escoria las cuales se dan de la siguiente manera:

- Etapa de fusión, es donde se eliminan las impurezas del acero líquido de manera química insertando manganeso, silicio, carbono, cal y oxígeno, generando la escoria espumante, la cual se desecha al final de este proceso.

- Etapa de afino, es la que se realiza después del proceso de fundición, en esta etapa se busca eliminar el resto de impurezas o añadir componentes que permita obtener un acero líquido conforme a la norma establecida de calidad. Durante este proceso se forma la escoria blanca.

Para el caso de la escoria negra, en el horno de arco eléctrico se tiene un rendimiento metálico de $87 \%$, eso quiere decir que de cada 100 toneladas de chatarra que ingresan para fundición, se generan 87 toneladas de acero líquido y 13 toneladas de escoria negra, se estima que en el año 2016 ANDEC generó un desperdicio aproximado de 22.000 toneladas de escoria negra.

Al finalizar el proceso de fundición, la escoria negra se la descarga desde el horno de arco eléctrico en una zona exclusiva para este material, de ahí es reubicado cerca de la misma zona por una excavadora para que se enfríe, ya enfriada se la trasladada al patio de chatarra donde se forma un montículo para que sea retirada por la empresa Puerto Limpio en el caso de ANDEC, la cual está encargada de la recolección de basura y desechos de la empresa.

Las empresas siderúrgicas en la actualidad consideran la escoria como material de desecho del proceso de producción, por lo cual gestiona con las empresas de limpieza el retiro de la misma de sus instalaciones. Según Pérez(2012) es importante aprovechar el uso de este residuo a través de usar tecnología y/o estrategias que permiten su utilización óptima, En el Ecuador las empresas que generar este material son ANDEC, ADELCA y NOVACERO, las cuales utilizan lo utilizan como relleno en algunos sectores de sus predios (previo un proceso de tratamiento básico de la misma), debido a que este material es poroso y no absorbe agua, lo cual evita que se formen superficies lodosas. (López, 2011) 
La composición química de la escoria negra está constituida de entre 24 - 35\% de Óxidos de Hierro, el cual puede ser recuperado a través de un proceso de fragmentación y ser reutilizada en la fundición de chatarra, el restante debe ser clasificado según por fracciones granulométricas (Vázquez, s.f.). La escoria negra puede ser utilizada en:

- Sector de construcción: materia prima de cemento y bloques de hormigón, aditivo para asfaltos, rellenos sanitarios, balastro ferroviario, gaviones de piedra y/o base para caminos.

- Sector agrícola: fertilizante, cubierta de suelo para control de la erosión y/o filtros de agua granulares

Para la escoria blanca ya se ha implementado un proceso de reciclaje de la misma para recuperar la cal, para ser reutilizada en el proceso de fundición de la chatarra. Según la National Slag Association, después de los estudios respectivos, la escoria ha sido declarada como como material no peligroso para el ambiente bajo los estándares de la EPA (Environmental Protection Agency), lo cual lo hace recomendable para su uso en las distintas formas que se han nombrado anteriormente (Lewis, 1980).

\section{MATERIALES Y MÉTODOS}

La presentes investigación cuenta con dos fases, para la fase cualitativa como refiere (Hernández, Fernández, \& Baptista (2014) se desea conocer y obtener información que se encuentre asociada a conocimientos, percepciones, actitudes, requerimientos y experiencias sobre el uso de escoria negra como materia prima para cemento, bloques y hormigón, este proceso de recolección se lo realizara a través de la aproximación y discusión sobre la temática con la participación de personas interesadas en el tema y que han ejercido práctica en la fabricación de los productos anteriormente descritos, todo este proceso será registrado para su posterior desgravación de forma literal.

La escoria negra presenta algunas características interesantes como elevada dureza, gran peso específico y una gran resistencia al desgaste, a través de un proceso de recuperación y valorización, para ser convertido en árido siderúrgico que puede ofrecer mejores prestaciones y sostenibilidad comparado a los áridos naturales (PROMSA).

Objetivos específicos y descripción de la información que se espera conseguir:

- Describir los requerimientos técnicos que debe cumplir la escoria negra para poder ser aceptada por el cliente, de esta manera se podrá definir el alcance del proceso a realizarse en la escoria negra para su comercialización.

- Determinar la frecuencia y cantidades promedios de escoria requerida para las aplicaciones en el sector de la construcción, esta información será útil para comprobar si la cantidad producida de escoria a nivel nacional será suficiente para cubrir las necesidades de los clientes.

- Definir un precio aproximado a pagar por la escoria negra una vez que cumpla con los requerimientos técnicos solicitados, además de comparar con los precios de las materias primas que se busca sustituir y si el precio es un factor determinante para la adquisición de las mismas.

Para obtener esta información se organizó un grupo focal, el cual estuvo compuesto de 7 personas, las cuales se encuentren trabajando en empresas de fabricación de bloques, hormigón o cemento, para esta reunión se va a realizar una sesión en un lugar neutral en la cual se van a tratar los temas de interés dirigidos por un moderador que se encargue de conducir la discusión y mantener la atención de los participantes. 
1. Investigación concluyente descriptiva

\section{ENCUESTA DE ÁRIDOS EN LA CONSTRUCCIÓN}

Para la presente investigación se realizará una encuesta a fin de verificar datos e información obtenidos de la investigación exploratoria, además obtener información que nos permita concluir sobre la aceptación del producto en el sector de la construcción dado que el uso de este producto no es muy común en la construcción.

La encuesta va dirigida a los profesionales relacionados al sector de la construcción al cual estará dirigido el producto, entre el perfil de los encuestados deberán ser Ingenieros Civiles, arquitectos o Maestros de obras. Para este tipo de encuesta no aplica el uso de recompensas, debido a que el tipo de producto no lo amerita. Las herramientas para apoyo de las encuestas se tendrán fotografías que permitan identificar el producto, las cuales se encuentran adjuntas en la encuesta.

\section{Definición de la Población Meta}

El grupo objetivo al cual irá dirigida la Encuesta serán las personas relacionadas con el Sector de la Construcción. El elemento del grupo objetivo, deberá tener un conocimiento básico acerca de los materiales que se utilizan en el Sector de la Construcción. La unidad de muestreo, para este caso son los ingenieros civiles, arquitectos, maestros de obras y personas afines al Sector de la Construcción, residentes o que estén ejerciendo su profesión en la Ciudad de Guayaquil.

\section{Determinación del Marco muestral}

Para el Marco Muestral se tomarán en cuenta a los elementos que se encuentren afiliados al Colegio del Ingenieros Civiles del Guayas o a la Cámara de Construcción de Guayaquil, además también también se considerarán personas que ejerzan su profesión de manera independiente y estén relacionados al Sector de la Construcción.

\section{Selección de la Técnica de Muestreo}

Para el presente trabajo se empleará una técnica de Muestreo No Probabilística por Conveniencia, esto debido a que al ser un grupo objetivo que debe cumplir con una especialidad no tan común, resulta complicado tener un fácil acceso a los elementos de la muestra, por tal motivo se buscará acceder a la mayor cantidad de elementos posibles.

\section{Definición del Tamaño de la Muestra}

Para definir el tamaño total de la Población Objetivo, se indagó la información disponible del Marco Muestral, de la cual solo se pudo obtener una referencia de la cantidad de Ingenieros Civiles afiliados a los Colegios del Ecuador. Esta información se la obtuvo de la página del Colegio de Ingenieros Civiles del Ecuador en la cual constan los afiliados en los Colegios de todas las Provincias.se obtuvo información de la página del Colegio de Ingenieros Civiles del Ecuador.

Gracias al uso de la herramienta Rasoft, se obtuvo el tamaño de la muestra:

N (población): 13088 ingenieros

Margen de error: $\quad 5 \%$

Confianza: $\quad 95 \%$

Distribución: $\quad 50 \%$ 


\section{MUESTRA POBLACIONAL: $\quad 374$ ingenieros}

6. Trabajo de campo: alcance Geográfico, días de duración.

La encuesta estará dirigida a la ciudad de Guayaquil y se estima que bastará un tiempo de 2 semanas para la recolección de la información.

\section{RESULTADOS Y DISCUSIÓN}

- Identificando el uso principal de la escoria para el sector de la construcción, la misma puede servir como sustituto de los áridos naturales, que sirven, según se tiene comprobado, para el mejoramiento de suelo, aditivo para la elaboración de hormigón y también se le ha intentado como materia prima para el cemento.

- Además, se puede observar que para el sector de la construcción los principales usos de los áridos según la importancia quedo en el siguiente orden:
1. Relleno
2. Hormigón
3. Mortero
4. Capa de Rodadura
5. Bases y Sub-bases
6. Colleras

\section{Tabla 1}

\section{Estadísticos}

\begin{tabular}{|c|c|c|c|c|c|c|c|}
\hline \multicolumn{8}{|c|}{ Estad } \\
\hline & & $\begin{array}{l}\text { Uso árido } \\
\text { en relleno }\end{array}$ & $\begin{array}{l}\text { Uso árido } \\
\text { hormigón }\end{array}$ & $\begin{array}{l}\text { Uso árido } \\
\text { Morteros }\end{array}$ & $\begin{array}{c}\text { Uso árido } \\
\text { Capas } \\
\text { rodadura }\end{array}$ & $\begin{array}{c}\text { Uso árido } \\
\text { Base y sub } \\
\text { base }\end{array}$ & $\begin{array}{l}\text { Uso árido } \\
\text { Collera }\end{array}$ \\
\hline $\mathrm{N}$ & Válido & 51 & 51 & 51 & 51 & 51 & 51 \\
\hline & Perdidos & 0 & 0 & 0 & 0 & 0 & 0 \\
\hline Moda & & 1 & 2 & 3 & 4 & 5 & 6 \\
\hline Rango & & 5 & 5 & 5 & 4 & 4 & 5 \\
\hline Mínimo & & 1 & 1 & 1 & 2 & 2 & 1 \\
\hline Máximo & & 6 & 6 & 6 & 6 & 6 & 6 \\
\hline Suma & & 107 & 110 & 176 & 214 & 210 & 254 \\
\hline
\end{tabular}

- Según las primicias para el uso principal de la escoria, se puede apuntar como principales clientes a quienes estén relacionados con el sector de la construcción, es decir a los responsables de ejecución de obras y además para los fabricantes de cemento.

- Identificado los clientes potenciales se determinó la frecuencia y cantidades aproximadas que se necesitan, esto va a depender según del tipo de construcción que se esté realizando, por ejemplo, 
para la construcción del nuevo hospital de IESS en Los Ceibos se estima que se utilizaron por lo menos 116 mil metros cuadrados de áridos.

- Con base en los datos obtenidos se puede apreciar que los Ingenieros Civiles, son los profesionales que usan más diferentes tipos de áridos, que, al resto, tal como se muestra en la siguiente tabla:

Tabla 2

Tabla cruzada Tipo de árido que usa con más frecuencia

\begin{tabular}{|c|c|c|c|c|c|c|c|}
\hline & & & \multicolumn{4}{|c|}{ Tipo de árido que ha usado con más frecuencia } & \multirow[b]{2}{*}{ Total } \\
\hline & & & $\begin{array}{c}\text { Áridos } \\
\text { naturales } \\
\text { (Calizos) }\end{array}$ & $\begin{array}{c}\text { Áridos } \\
\text { Reciclados } \\
\text { (procesados } \\
\text { de } \\
\text { demoliciones } \\
\text { ) }\end{array}$ & $\begin{array}{c}\text { Áridos } \\
\text { Artificiales } \\
\text { (siderúrgicos) }\end{array}$ & Desconoce & \\
\hline \multirow[t]{16}{*}{ profesion del individuo } & \multirow[t]{4}{*}{ Arquitecto } & Recuento & 12 & 2 & 1 & 2 & 17 \\
\hline & & $\begin{array}{l}\% \text { dentro de profesion del } \\
\text { individuo }\end{array}$ & $70,6 \%$ & $11,8 \%$ & $5,9 \%$ & $11,8 \%$ & $100,0 \%$ \\
\hline & & $\begin{array}{l}\text { \% dentro de Tipo de árido } \\
\text { que ha usado con más } \\
\text { frecuencia }\end{array}$ & $40,0 \%$ & $28,6 \%$ & $20,0 \%$ & $22,2 \%$ & $33,3 \%$ \\
\hline & & $\%$ del total & $23,5 \%$ & $3,9 \%$ & $2,0 \%$ & $3,9 \%$ & $33,3 \%$ \\
\hline & \multirow[t]{4}{*}{ Ingeniero Civil } & Recuento & 15 & 2 & 3 & 1 & 21 \\
\hline & & $\begin{array}{l}\% \text { dentro de profesion del } \\
\text { individuo }\end{array}$ & $71,4 \%$ & $9,5 \%$ & $14,3 \%$ & $4,8 \%$ & $100,0 \%$ \\
\hline & & $\begin{array}{l}\text { \% dentro de Tipo de árido } \\
\text { que ha usado con más } \\
\text { frecuencia }\end{array}$ & $50,0 \%$ & $28,6 \%$ & $60,0 \%$ & $11,1 \%$ & $41,2 \%$ \\
\hline & & $\%$ del total & $29,4 \%$ & $3,9 \%$ & $5,9 \%$ & $2,0 \%$ & $41,2 \%$ \\
\hline & \multirow[t]{4}{*}{ Maestro de Obra } & Recuento & 1 & 2 & 1 & 4 & 8 \\
\hline & & $\begin{array}{l}\% \text { dentro de profesion del } \\
\text { individuo }\end{array}$ & $12,5 \%$ & $25,0 \%$ & $12,5 \%$ & $50,0 \%$ & $100,0 \%$ \\
\hline & & $\begin{array}{l}\text { \% dentro de Tipo de árido } \\
\text { que ha usado con más } \\
\text { frecuencia }\end{array}$ & $3,3 \%$ & $28,6 \%$ & $20,0 \%$ & $44,4 \%$ & $15,7 \%$ \\
\hline & & $\%$ del total & $2,0 \%$ & $3,9 \%$ & $2,0 \%$ & $7,8 \%$ & $15,7 \%$ \\
\hline & \multirow[t]{4}{*}{ Otro } & Recuento & 2 & 1 & 0 & 2 & 5 \\
\hline & & $\begin{array}{l}\% \text { dentro de profesion del } \\
\text { individuo }\end{array}$ & $40,0 \%$ & $20,0 \%$ & $0,0 \%$ & $40,0 \%$ & $100,0 \%$ \\
\hline & & $\begin{array}{l}\text { \% dentro de Tipo de árido } \\
\text { que ha usado con más } \\
\text { frecuencia }\end{array}$ & $6,7 \%$ & $14,3 \%$ & $0,0 \%$ & $22,2 \%$ & $9,8 \%$ \\
\hline & & $\%$ del total & $3,9 \%$ & $2,0 \%$ & $0,0 \%$ & $3,9 \%$ & $9,8 \%$ \\
\hline \multirow[t]{4}{*}{ Total } & & Recuento & 30 & 7 & 5 & 9 & 51 \\
\hline & & $\begin{array}{l}\% \text { dentro de profesion del } \\
\text { individuo }\end{array}$ & $58,8 \%$ & $13,7 \%$ & $9,8 \%$ & $17,6 \%$ & $100,0 \%$ \\
\hline & & $\begin{array}{l}\text { \% dentro de Tipo de árido } \\
\text { que ha usado con más } \\
\text { frecuencia }\end{array}$ & $100,0 \%$ & $100,0 \%$ & $100,0 \%$ & $100,0 \%$ & $100,0 \%$ \\
\hline & & $\%$ del total & $58,8 \%$ & $13,7 \%$ & $9,8 \%$ & $17,6 \%$ & $100,0 \%$ \\
\hline
\end{tabular}

Gracias a la prueba de Chi-Cuadrado se puede decir que existe independencia (Sig. $=0,88)$, entre las variables relacionadas en la Tabla Cruzada, por lo que el uso del árido es indiferente al profesional que lo use.

- Además, para la empresa HOLCIM, productora de cemento, produce 300 mil toneladas de cemento de manera mensual, siendo el cemento un producto elaborado $100 \%$ con áridos naturales, de lo cual solo el $15 \%$ se lo adquiere a otras empresas, eso significa que busca en el mercado mensualmente de 45 mil toneladas de áridos, lo cual sería un total de 540 mil toneladas anuales.

- Para el uso de la escoria para aplicaciones en la construcción esta debe estar regulada según la normativa dispuesta para su uso en la construcción, esto se debe avalar mediante pruebas de laboratorio que demuestren el índice de plasticidad, grado de resistencia, propiedades físicas y químicas, además de que se le debe aplicar un proceso de molido y tamizado para que obtener diferentes escorias con tamaño de grano que requiere las aplicaciones de la construcción y 
fabricación de otros productos. De igual manera, la escoria para ser usada como materia prima para el cemento, bloques u hormigón, ha esta debe realizarse pruebas de laboratorio.

- Para que la escoria sea un sustituto valido para los clientes se debe tomar en cuenta el precio, el cual es un parámetro muy importante en el sector de la construcción y de la producción, por lo que se lo va a comparar con el precio del ripio (grava o cascajo) por metro cúbico que según el uso varía entre 4,94 a 5,06 dólares, valor incluido con puesto en obra, el cual se obtiene de las diferentes canteras que se encuentras a las afueras de la ciudad. De este valor también incluye el proceso de triturado y tamizado que es el proceso normal para los áridos.

- Los precios dados por los áridos naturales, es el que se encuentra dado en el mercado, se desconoce los descuentos que se den para clientes frecuentes.

- Para la forma de pago, por lo visto difiere del tiempo establecido en el negocio del constructor ya que el quien tiene varios años de trayectoria puede conseguir el árido puesto en obra y ha crédito hasta que se le sea cancelado por la obra que se encuentra trabajando.

- Para el caso como materia prima del cemento, en el caso de HOLCIM dentro de su proceso productivo incluye la obtención y procesamiento de materia prima desde 3 cerros que se encuentran dentro de sus instalaciones, por lo que ayuda a dinamizar la disponibilidad de este producto.

- Con referencia a los canales de distribución, debido a que la preferencia de la constructoras y empresas productoras es que el producto sea puesto en obra, sería necesario contratar una flota para el transporte de la escoria hacia el lugar que prefiera el cliente, dicho valor de transporte no se lo debe cobrar al cliente, sino que se lo debe asumir.

Todos los encuestados, prefieren que el producto sea entregado en la obra, tal como indica las siguientes tablas:

Tabla 3

Manera en qué ha recibido el árido

Manera en que ha recibido el árido

\begin{tabular}{|c|c|c|c|c|c|}
\hline & & Frecuencia & Porcentaje & $\begin{array}{c}\text { Porcentaje } \\
\text { válido }\end{array}$ & $\begin{array}{l}\text { Porcentaje } \\
\text { acumulado }\end{array}$ \\
\hline \multirow[t]{4}{*}{ Válido } & Puesto en Obra & 21 & 41,2 & 41,2 & 41,2 \\
\hline & Tiene que ir a ver & 24 & 47,1 & 47,1 & 88,2 \\
\hline & Desconoce & 6 & 11,8 & 11,8 & 100,0 \\
\hline & Total & 51 & 100,0 & 100,0 & \\
\hline
\end{tabular}

Tabla 4

Manera en qué le gustaría recibir el producto

Manera que le gustaría recibir el producto

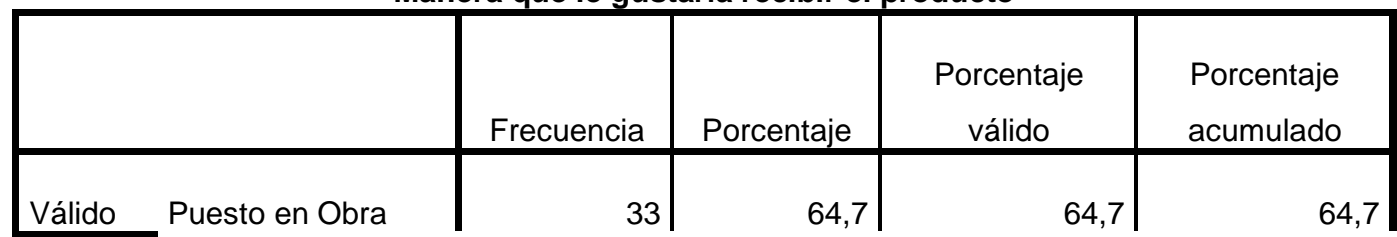




\begin{tabular}{|l|r|r|r|r|}
\hline Tiene que ir a ver & 9 & 17,6 & 17,6 & 82,4 \\
Le es indiferente & 9 & 17,6 & 17,6 & 100,0 \\
Total & 51 & 100,0 & 100,0 & \\
\hline
\end{tabular}

- Debido a lo mencionado anteriormente, el cliente para adquirir un producto sustituto de los áridos estaría dispuesto a pagar el mismo o menor precio.

- En referencia al uso de escoria para la producción de cemento, esta ya habría sido usada anteriormente para la elaboración de este producto, sin embargo, presentó problemas por el elevado porcentaje de contenido de hierro que afectaba a las maquinarias en el proceso productivo, por lo que sería necesario aplicar un un proceso de tratamiento a la escoria para extraer el excedente de materiales ferrosos a la misma, por lo cual aumentaría su costo.

- Para el caso de bloques de hormigón, de lo que se tiene entendido es que, para utilizar a la escoria como aditivo para cualquier aplicación del hormigón, es necesario realizar pruebas de laboratorio, las cuales se han realizado en tesis pero que no han sido difundidos hacía los interesados.

- Asimismo, para el uso de escoria como aditivo de asfalto, rellenos sanitarios y base para caminos, aplica de igual manera al literal anterior, y cabe recalcar que en otros países la escoria ya está siendo utilizada como aditivo para la construcción.

- Por lo obtenido de las encuetas, las propiedades más importantes tienen el siguiente orden:

1. Propiedades mecánicas y físicas

2. Propiedades geométricas (empata en el primer lugar)

3. Propiedades térmicas y de alteración

4. Propiedades químicas

Tabla 5

Estadísticos

Estadísticos

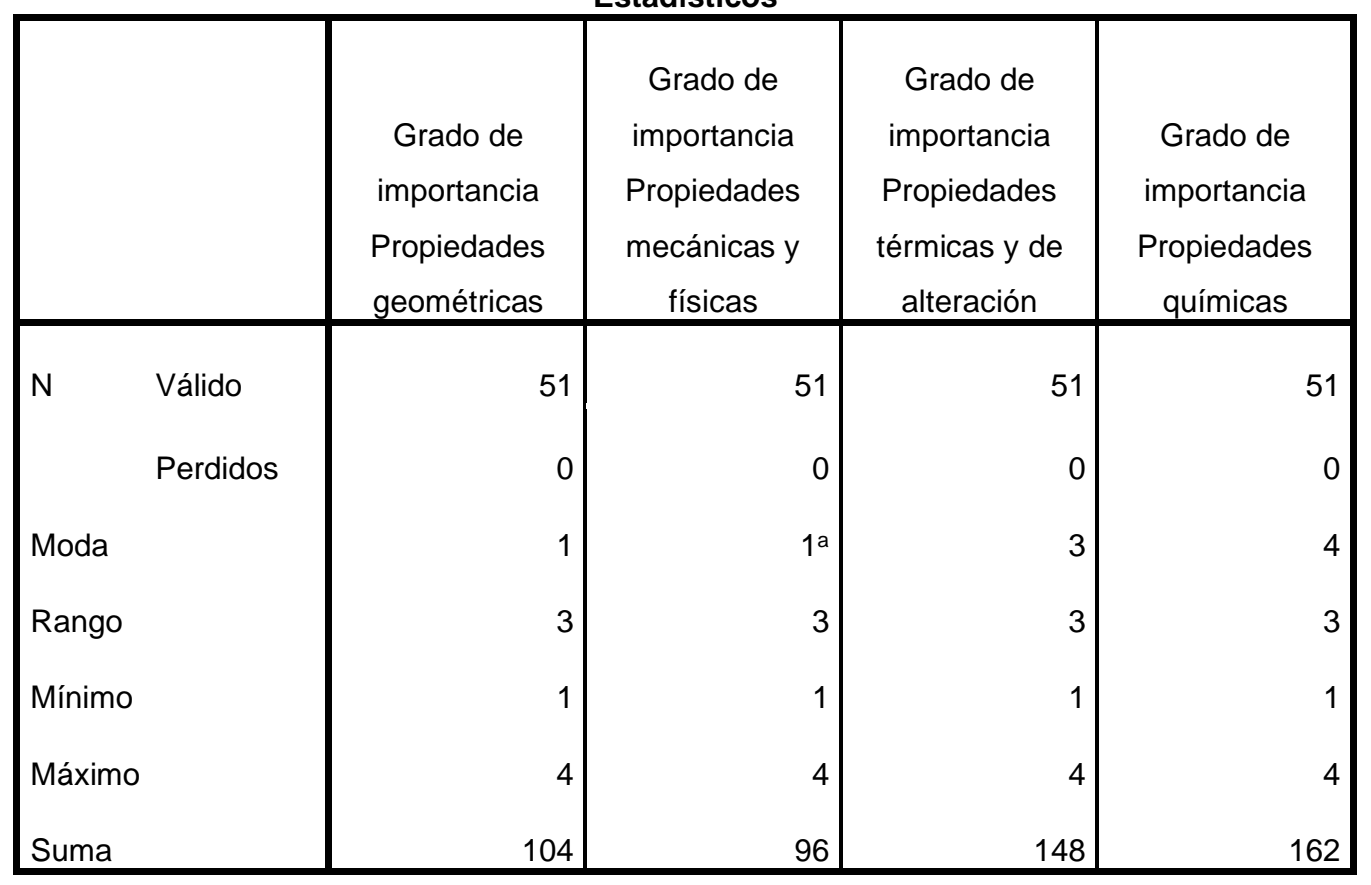

a. Existen múltiples modos. Se muestra el valor más pequeño. 
- $\mathrm{Al}$ presente no existen empresas que utilicen la escoria como materia prima, se tiene conocimiento de su uso para casos aislados, pero son para obras menores o adecuaciones.

- En el sector de la construcción una de las desventajas que se nos indica es que antes de ser usados los aridos deben de cumplir con la normativa, tal como es el caso de la plasticidad, por lo que para evitar esté inconveniente se le agrega arena o cemento a los áridos.

- En cambio, para la fabricación no tiene ninguna desventaja utilizar el árido natural, ya que tiene un riguroso control de calidad que se encarga de revisar la granulometría y humedad, de ser el caso de no cumplir con la calidad requerida puede ser reprocesado y reemplazado de forma inmediata.

- Para los dos casos es que los áridos son un producto ya establecido en el mercado y en la mente de los consumidores, que son escépticos a cambios según la forma tradicional, además de eso

- Actualmente en el Ecuador existen tres fundidoras de chatarras, las cuales son ANDEC, ADELCA y NOVACERO, entre las tres se ha logrado producir un total aproximado de 500 mil toneladas de acero fundido, de dicho proceso se sabe que en este tipo de proceso se tiene en promedio un rendimiento metálico del $87 \%$, del cual el 13\% corresponde a escoria, por lo tanto, al producir las 500 mil toneladas de acero también se produjeron como desperdicio aproximadamente 74,7 mil toneladas de escoria.

- De lo consultado se pudo encontrar que la NSA (National Slag Association - Asociación Nacional de Escoria, Estados Unidos), realizo pruebas para determinar la peligrosidad de la escoria con el medio ambiente, de lo cual se había concluido que la escoria no es un material dañino para el medio ambiente y seres vivos.

- De igual manera, la escoria es un material que no reacciona ni produce de manera significativa ningún elemento dañino, no se conoce de otra sustancia que reaccione con la escoria.

- Como ventajas, para su uso en la construcción, se tiene experiencia que tiene un alto índice de plasticidad, mayor al de los áridos naturales, daría excelentes resultados en la aplicación de mejoramiento de suelos o relleno, además que es un buen material filtrante, siendo esto útil para cimentaciones para evitar el empozamiento de líquidos, sin embargo para esta aplicación sería necesaria también la aplicación de una sub-base para que impermeabilice, es decir que impida que la base de la cimentación pueda entrar agua, volviéndolo un terreno lodoso, también sirve para ser utilizado como base y sub-base para caminos y carreteras, debido a la geometría de la escoria que es rugosa, al momento de ser regada en el piso, esto hace que se adhieran unos con otros y de esta forma cuando se la aplane quede fijado como un solo bloque. Sin embargo, todas estas ventajas deben ser comprobadas con las respectivas pruebas de laboratorio que avalen dichas propiedades. En cambio, de las desventajas se podría decir que la cantidad que se produce nacionalmente de escoria en el país no sería suficiente para cubrir la demanda que constituiría una obra como la del Hospital del IESS en Los Ceibos, por lo que se debe enfocar en obras menores o adecuaciones.

- Según el tipo de construcción se puede apreciar que el uso de áridos según su aplicación varia, tal como se muestra en la siguiente tabla: 
Tabla 6

Tabla Cruzada Mayor uso en el campo con Material de Relleno

\begin{tabular}{|c|c|c|c|c|c|c|c|c|}
\hline & & & \multicolumn{5}{|c|}{ Mayor uso en el campo con Material de relleno } & \multirow[b]{2}{*}{ Total } \\
\hline & & & 1 & 2 & 3 & 4 & 5 & \\
\hline \multirow{24}{*}{$\begin{array}{l}\text { Campo de la } \\
\text { construcción }\end{array}$} & \multirow[t]{4}{*}{0} & Recuento & 0 & 0 & 0 & 0 & 1 & 1 \\
\hline & & $\begin{array}{l}\text { \% dentro de Campo de la } \\
\text { construcción }\end{array}$ & $0,0 \%$ & $0,0 \%$ & $0,0 \%$ & $0,0 \%$ & $100,0 \%$ & $100,0 \%$ \\
\hline & & $\begin{array}{l}\text { \% dentro de Mayor uso } \\
\text { en el campo con Material } \\
\text { de relleno }\end{array}$ & $0,0 \%$ & $0,0 \%$ & $0,0 \%$ & $0,0 \%$ & $9,1 \%$ & $2,2 \%$ \\
\hline & & $\%$ del total & $0,0 \%$ & $0,0 \%$ & $0,0 \%$ & $0,0 \%$ & $2,2 \%$ & $2,2 \%$ \\
\hline & \multirow{4}{*}{$\begin{array}{l}\text { Construcciones } \\
\text { residencial }\end{array}$} & Recuento & 1 & 3 & 3 & 6 & 5 & 18 \\
\hline & & $\begin{array}{l}\% \text { dentro de Campo de la } \\
\text { construcción }\end{array}$ & $5,6 \%$ & $16,7 \%$ & $16,7 \%$ & $33,3 \%$ & $27,8 \%$ & $100,0 \%$ \\
\hline & & $\begin{array}{l}\% \text { dentro de Mayor uso } \\
\text { en el campo con Material } \\
\text { de relleno }\end{array}$ & $14,3 \%$ & $60,0 \%$ & $30,0 \%$ & $50,0 \%$ & $45,5 \%$ & $40,0 \%$ \\
\hline & & $\%$ del total & $2,2 \%$ & $6,7 \%$ & $6,7 \%$ & $13,3 \%$ & $11,1 \%$ & $40,0 \%$ \\
\hline & \multirow{4}{*}{$\begin{array}{l}\text { Construcciones } \\
\text { comerciales }\end{array}$} & Recuento & 2 & 1 & 3 & 2 & 2 & 10 \\
\hline & & $\begin{array}{l}\text { \% dentro de Campo de la } \\
\text { construcción }\end{array}$ & $20,0 \%$ & $10,0 \%$ & $30,0 \%$ & $20,0 \%$ & $20,0 \%$ & $100,0 \%$ \\
\hline & & $\begin{array}{l}\text { \% dentro de Mayor uso } \\
\text { en el campo con Material } \\
\text { de relleno }\end{array}$ & $28,6 \%$ & $20,0 \%$ & $30,0 \%$ & $16,7 \%$ & $18,2 \%$ & $22,2 \%$ \\
\hline & & $\%$ del total & $4,4 \%$ & $2,2 \%$ & $6,7 \%$ & $4,4 \%$ & $4,4 \%$ & $22,2 \%$ \\
\hline & \multirow{4}{*}{$\begin{array}{l}\text { Construcciones } \\
\text { industriales }\end{array}$} & Recuento & 1 & 1 & 1 & 3 & 0 & 6 \\
\hline & & $\begin{array}{l}\text { \% dentro de Campo de la } \\
\text { construcción }\end{array}$ & $16,7 \%$ & $16,7 \%$ & $16,7 \%$ & $50,0 \%$ & $0,0 \%$ & $100,0 \%$ \\
\hline & & $\begin{array}{l}\text { \% dentro de Mayor uso } \\
\text { en el campo con Material } \\
\text { de relleno }\end{array}$ & $14,3 \%$ & $20,0 \%$ & $10,0 \%$ & $25,0 \%$ & $0,0 \%$ & $13,3 \%$ \\
\hline & & $\%$ del total & $2,2 \%$ & $2,2 \%$ & $2,2 \%$ & $6,7 \%$ & $0,0 \%$ & $13,3 \%$ \\
\hline & \multirow{4}{*}{$\begin{array}{l}\text { Construcciones } \\
\text { institucionales }\end{array}$} & Recuento & 0 & 0 & 2 & 1 & 2 & 5 \\
\hline & & $\begin{array}{l}\text { \% dentro de Campo de la } \\
\text { construcción }\end{array}$ & $0,0 \%$ & $0,0 \%$ & $40,0 \%$ & $20,0 \%$ & $40,0 \%$ & $100,0 \%$ \\
\hline & & $\begin{array}{l}\% \text { dentro de Mayor uso } \\
\text { en el campo con Material } \\
\text { de relleno }\end{array}$ & $0,0 \%$ & $0,0 \%$ & $20,0 \%$ & $8,3 \%$ & $18,2 \%$ & $11,1 \%$ \\
\hline & & $\%$ del total & $0,0 \%$ & $0,0 \%$ & $4,4 \%$ & $2,2 \%$ & $4,4 \%$ & $11,1 \%$ \\
\hline & \multirow{4}{*}{$\begin{array}{l}\text { Construcción de obras } \\
\text { publicas }\end{array}$} & Recuento & 3 & 0 & 1 & 0 & 1 & 5 \\
\hline & & $\begin{array}{l}\text { \% dentro de Campo de la } \\
\text { construcción }\end{array}$ & $60,0 \%$ & $0,0 \%$ & $20,0 \%$ & $0,0 \%$ & $20,0 \%$ & $100,0 \%$ \\
\hline & & $\begin{array}{l}\text { \% dentro de Mayor uso } \\
\text { en el campo con Material } \\
\text { de relleno }\end{array}$ & $42,9 \%$ & $0,0 \%$ & $10,0 \%$ & $0,0 \%$ & $9,1 \%$ & $11,1 \%$ \\
\hline & & $\%$ del total & $6,7 \%$ & $0,0 \%$ & $2,2 \%$ & $0,0 \%$ & $2,2 \%$ & $11,1 \%$ \\
\hline \multirow[t]{4}{*}{ Total } & & Recuento & 7 & 5 & 10 & 12 & 11 & 45 \\
\hline & & $\begin{array}{l}\text { \% dentro de Campo de la } \\
\text { construcción }\end{array}$ & $15,6 \%$ & $11,1 \%$ & $22,2 \%$ & $26,7 \%$ & $24,4 \%$ & $100,0 \%$ \\
\hline & & $\begin{array}{l}\% \text { dentro de Mayor uso } \\
\text { en el campo con Material } \\
\text { de relleno }\end{array}$ & $100,0 \%$ & $100,0 \%$ & $100,0 \%$ & $100,0 \%$ & $100,0 \%$ & $100,0 \%$ \\
\hline & & $\%$ del total & $15,6 \%$ & $11,1 \%$ & $22,2 \%$ & $26,7 \%$ & $24,4 \%$ & $100,0 \%$ \\
\hline
\end{tabular}

De lo cual se puede concluir de basándonos en la prueba de Chi-Cuadrado, que existe independencia entre las dos variables comparadas en la Tabla Cruzada (Sig. $=0,464)$, por lo que es indiferente la aplicación del árido con el tipo de construcción que se realice.

\section{CONCLUSIÓN}

Como conclusión de la investigación, se puede decir que se debe crear una percepción para el uso de la escoria en el sector de la construcción y fabricación de materiales para el mismo sector, ya que solo se han efectuado obras menores con la escoria y estudios con poca difusión a los clientes potenciales, además para que la escoria pueda ingresar como un sustituto para los áridos, primero es necesario industrializar el proceso ya que se requiere de tratar al material para dar características únicas que requieren los clientes y además de someterla a varias pruebas de laboratorio para generar confiabilidad en el producto, como segundo punto se debería contar con una flota de transporte que permita dar un valor agregado visible para el cliente, que requiere el producto puesto en obra, debido 
a estos dos puntos es que se aumentaría el costo de venta de la escoria por lo que le sería difícil competir con los áridos naturales sino no se dispone de una gran fuente de este producto, para aprovechar de las economías de escala, el cual es otro inconveniente, ya que en el Ecuador se podrían producir un aproximado de 74,7 mil toneladas de escoria, lo cual es una cantidad muy pequeña para los requerimientos del sector constructivo.

Por lo tanto, se recomendaría el uso de la escoria para obras menores y adecuaciones dentro de la empresa, y generar proyectos sociales utilizando este producto sin fines de lucro, para el mejoramiento de calles, rellenos o construcciones menores para la comunidad, creando una percepción positiva dentro de la misma acerca del uso de la escoria para aplicaciones de la construcción.

\section{REFERENCIAS}

ANDEC. (5 de Enero de 2018). ANDEC. Obtenido de https://www.andec.com.ec/index.php/es/inicio

FEDIMETAL. (2014). Política, Estrategias y Plan de Acción 2035 para la Industria Metalmecánica Ecuatoriana. Construmetal , 13-14.

Hernández, R., Fernández, C., \& Baptista, P. (2014). Metodología de la Investigación. México D.F.: Mc Graw Hill Education.

Jiménez, M., \& Navarrete, M. (2018). Perfil ecuatoriano de la empresas metalmecánicas. Dominio de la Ciencias, IV(1), 585-602. doi:10.23857/dom.cien.pocaip.2017.4.1.enero.585-602

Lewis, D. (14 de Diciembre de 1980). NATIONAL SLAG ASSOCIATION . Obtenido de IRON and STEEL SLAG - A NON-HAZARD: http://www.nationalslag.org/sites/nationalslag/files/documents/nsa_1945_slag_a_non-hazard $\% 20 \% 281 \% 29 . p d f$

López, V. (2011). Aprovechamiento de escorias blancas (LFS) y negras (EAFS) de acería eléctrica de estabilización de suelos y en capas firmes del caminos rurales. Burgos.

Nicolalde, S. V. (2008). Utilización de escorias y polvos de acería en la producción de bloques y adoquines. Quito: EPN.

Pérez, A. M. (2012). Selección de tecnologías apropiadas para el aprovechamiento de la escoria en el sector siderúrgico (Doctoral dissertation, Universidad Tecnológica de Pereira. . Facultad de Ciencias Ambientales. Administración Ambiental.

PROMSA. (s.f.). Promsa. Obtenido de http://www.promsa.com/c/document_library/get_file?uuid=0b0820f253fd-40c2-bbc4-112101368fbf

Vázquez, E. (s.f.). UTILIZACIÓN DE ÁRIDOS SIDERÚRGICOS. Obtenido de http://www.arccat.net/es/publicacions/pdf/ccr/jornades_construccio/300105_p3.pdf 